\title{
Structural basis of the anti-ageing effects of polyphenolics: mitigation of oxidative stress
}

\author{
Adam Rolt(10 and Lynne S. Cox ${ }^{*}$ (1)
}

\begin{abstract}
Ageing, and particularly the onset of age-related diseases, is associated with tissue dysfunction and macromolecular damage, some of which can be attributed to accumulation of oxidative damage. Polyphenolic natural products such as stilbenoids, flavonoids and chalcones have been shown to be effective at ameliorating several age-related phenotypes, including oxidative stress, inflammation, impaired proteostasis and cellular senescence, both in vitro and in vivo. Here we aim to identify the structural basis underlying the pharmacology of polyphenols towards ROS and related biochemical pathways involved in age-related disease. We compile and describe SAR trends across different polyphenol chemotypes including stilbenoids, flavonoids and chalcones, review their different molecular targets and indications, and identify common structural ground between chemotypes and mechanisms of action. In particular, we focus on the structural requirements for the direct scavenging of reactive oxygen/nitrogen species such as radicals as well as coordination of a broader antioxidant response. We further suggest that it is important to consider multiple (rather than single) biological activities when identifying and developing new medicinal chemistry entities with utility in modulating complex biological properties such as cell ageing.
\end{abstract}

Keywords: Ageing, Aging, Oxidative stress, Inflammation, Antioxidants, Polyphenols, Stilbenoids, Flavonoids, Chalcones, NRF2

\section{Background \\ Biological ageing and the potential of polyphenols as anti-ageing therapeutics}

Major demographic and clinical challenges arise from the rapid increase in the number of people aged over 65 years; such individuals are at risk of developing multiple age-related diseases [1]. The free radical theory of ageing, mooted over 60 years ago [2], suggests that reactive oxygen species (ROS), generated metabolically within eukaryotic cells or from exogenous sources [3], cause macromolecular damage which is detrimental to health. While the importance of free radicals to ageing phenotypes has been widely questioned [4], the urgent need to identify new drug-like molecules that can

*Correspondence: lynne.cox@bioch.ox.ac.uk Department of Biochemistry, University of Oxford, South Parks Road, Oxford OX1 3QU, UK modulate the causes of age-related diseases $[5,6]$ has led to a resurgence of interest in anti-oxidants, particularly natural products and their derivatives with polypharmacological properties.

Polyphenolic organic compounds were originally identified as natural products synthesized by plants to serve as pigments, anti-microbial agents (phytoalexins), and in signalling changes in plant state (e.g. growth, ripening) [7]. Their presence in foods associated with human health and longevity benefits, such as fruits and vegetables, tea, cocoa beans and olives, is suggestive that they may play an important dietary role in humans [8]. Understanding the mechanistic basis for health benefits, particularly in prevention and/or treatment of inflammatory and/or age-related diseases, i.e. how disparate molecular structures lead to overlapping phenotypic impacts, requires systematic analysis of classes of polyphenols so 
that structure-activity relationships (SAR) can be made clear.

Polyphenolic natural products such as stilbenoids, flavonoids and chalcones possess a varied and interesting pharmacological profile marked by interactions with a broad range of biological targets, as well as target-decoupled structurally-derived pharmacology [9-12]. Polyphenolic compounds have been shown to modulate the redox status of cells, to alter cellular signalling and to help prevent the accumulation of damage in long-lived biological molecules such as lipids, proteins and nucleic acids. This is accomplished both directly, through scavenging of reactive oxygen species, and indirectly, via interaction with transcription factors which coordinate the antioxidant response. Additionally, polyphenols can damp down inflammatory signalling, modulate nutrient sensing pathways, and induce the selective apoptosis of senescent cells. Importantly, these biological processes become dysfunctional with age and are causative in the pathogenesis of age-related disease [5, 6, 13, 14]. Hence there is renewed interest in developing polyphenol scaffolds and chemotypes as new therapeutics to ameliorate age-related disease.

Deciphering the precise molecular mechanisms of action of polyphenols in altering biological phenotypes of age-related disease is challenging due to the complexity of biological systems, where multiple different biochemical pathways can all contribute to a particular phenotypic outcome such as ageing. Here we aim to identify the structural basis underlying the pharmacology of polyphenols towards oxidative stress and related biochemical pathways involved in age-related disease. We compile and describe structure-activity relationship (SAR) trends across different polyphenol chemotypes including stilbenoids, flavonoids and chalcones, review their different molecular targets and indications, and identify common structural ground between chemotypes and mechanisms of action. In particular, we focus on the structural requirements for the direct scavenging of reactive oxygen/nitrogen species such as radicals as well as coordination of a broader antioxidant response.

\section{Main text}

\section{Polyphenols have direct anti-oxidant activity by reacting} with ROS to form stabilised radicals

Reactive oxygen species (ROS) such as hydroxyl/peroxyl radicals and peroxides are produced through the normal processes of metabolism, e.g. oxidative phosphorylation and ATP generation. Disrupted ROS homeostasis, which is observed with increasing biological age [3], can result either from increased ROS production or diminished ability to remove ROS. Both the source and nature of the reactive species are important in determining biological targets and the nature of ROS-mediated modifications. Notably, different ROS vary greatly in diffusion distances and reactivity [15]. While ROS can act as important signalling molecules [16, 17], they can also irreversibly damage critical macromolecules, including DNA [18]. Furthermore, ROS can induce cellular senescence both in vitro and in vivo through the p53-mediated DNA damage response $[19,20]$. Accordingly, sequestration of ROS has been demonstrated to improve health and even extend healthy lifespan in several model organisms [21-26].

Polyphenols can react with ROS in a stoichiometric manner to form stabilized radicals, following abstraction of hydrogen, as outlined in Fig. 1. Stabilisation of radicals through delocalisation over the stilbenoid and flavonoid scaffold increases the residence time and diffusion distance of the radicals, enabling stoichiometric clearance by endogenous antioxidants such as glutathione (GSH). As shown in Fig. 1, direct antioxidant activity thus depends on the presence of hydroxyl groups, which both provide a source of labile hydrogen for abstraction, and increase the stability of the radical formed.

\section{Key antioxidant features of resveratrol and other stilbenoids}

Resveratrol is arguably the most widely studied polyphenol in ageing science [9]. Resveratrol itself (entries 1a, 2a, $\mathbf{3 b}$ and $\mathbf{4 g}$, Fig. 2), cis-resveratrol $\mathbf{1 b}$ and derivatives $\mathbf{1 c}-$ If with varying degrees of methylation, all possess direct antioxidative capacity, as determined by an ABTS radical scavenging assay [27]. By contrast, fully substituted derivatives $\mathbf{1 g - 1 j}$, lacking critical hydroxyl groups, show greatly diminished or zero antioxidant capabilities [27].

The (E)-olefin functionality in resveratrol is important for potent radical scavenging activity since dihydro-resveratrol 1k, which lacks the trans-olefin double bond, was shown to have fivefold less potent direct antioxidant activity than resveratrol 1a, due to diminished ability to stabilise the radical via delocalisation (determined in a DPPH radical scavenging assay) [28, 29]. Furthermore, an alkynyl analogue of resveratrol $\mathbf{1 1}$ was found to be a less potent antioxidant than resveratrol $1 \mathbf{a}$ in a cell based model of oxidative stress [30], indicating requirement for the trans-olefin in resveratrol for potent antioxidant activity.

Given the critical role of a hydroxyl moiety in the formation of a stable stilbenoid radical (Fig. 1), it is therefore unsurprising that increasing the number of hydroxyl groups can increase the rate of radical scavenging. This could be attributed to the presence of ortho-OH groups, which also permit the scavenging of superoxide $\left(\mathrm{O}_{2}^{--}\right)$ radicals. In a series of six stilbenoids (Fig. 2 2a-2f), increasing in $\mathrm{OH}$ substitution from the tri-hydroxylated 

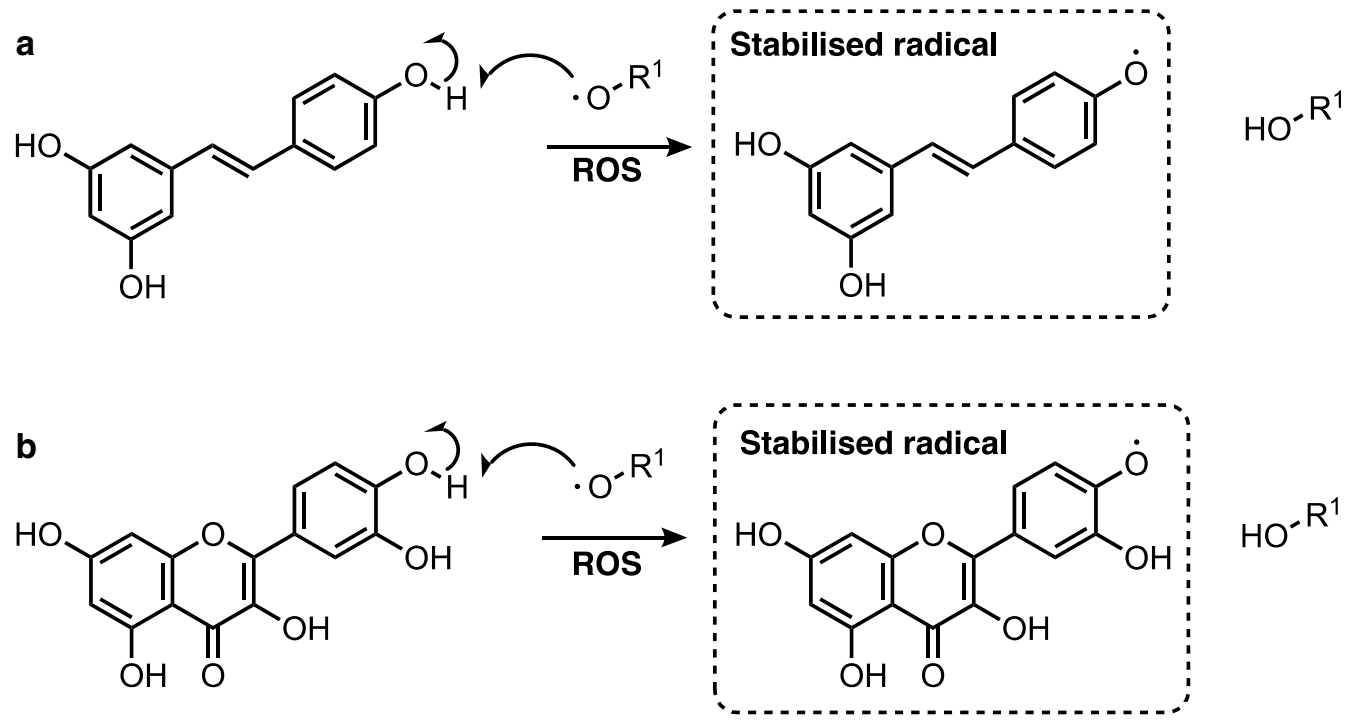

Fig. 1 Direct anti-oxidant activity of polyphenols: hydrogen abstraction, quenching of ROS and formation of stabilised stilbenoid (a) and flavonoid (b) radicals

resveratrol (entry 2a) up through to $3,3^{\prime}, 4,4^{\prime}, 5,5^{\prime}$-hexahydroxystilbene, $\mathbf{2 f}$ [31], it was demonstrated that the tetra substituted derivative $2 \mathrm{c}$ possessed inferior efficacy in a kinetic scavenging assay with DMPO radicals compared with resveratrol 2a. However entries $\mathbf{2 b}$ and $\mathbf{2 d}$ showed greatly improved activity, probably due to the relative substitution patterns, i.e. effect of neighbouring ortho$\mathrm{OH}$ groups, with second order rate constants for DPPH radical trapping in the order of $>10^{1}$ to $10^{3}$-fold higher than resveratrol 2a. Notably, compounds containing ortho- $\mathrm{OH}$ groups $\mathbf{2 b}, \mathbf{2 d - 2 f}$ were also able to scavenge superoxide $\left(\mathrm{O}_{2}^{--}\right)$radicals with low micromolar efficacy, whereas compounds lacking this ortho- $\mathrm{OH}$ functionality did not effectively scavenge $\mathrm{O}_{2}^{-}$. While possessing potent antioxidant activity in vitro, $\mathbf{2 b}$ and $\mathbf{2 d - 2 f}$ were also found to be approximately fourfold more toxic than resveratrol (2a) in human cells in culture (toxicity in

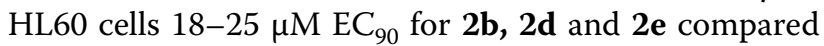
to $>100 \mu \mathrm{M}$ for resveratrol, 2a) [31], which could be due to the in situ formation of electrophilic quinone metabolites, as a consequence of increased radical scavenging.

The importance of the trans-olefin bond for resveratrol's antioxidant activity has been additionally investigated by isosteric replacement of the $\mathrm{C}=\mathrm{C}$ double bond in resveratrol for an imine, permitting the rapid synthesis of imine resveratrol analogues (IRAs), and evaluation of their biological activity (3a, Fig. 2). Twenty-five hydroxylated and methoxylated IRAs [32] were analysed for their ability to quench DPPH radicals and singlet oxygen. Compounds containing a hydroxyl group in the ' $\mathbf{B}$ ' ring possessed DPPH scavenging capacity $\left(\mathrm{EC}_{50}<1000 \mu \mathrm{M}\right)$, whereas derivatives with a hydroxyl group only present in ring ' $A$ ' did not $\left(E C_{50}>1000 \mu M\right)$. Structures 3c-3i, all possessing an ortho-hydroxyl group, were the most potent derivatives in terms of anti-oxidant activity in the $\mathrm{DPPH}$ radical scavenging assay $\left(\mathrm{EC}_{50} 10-30 \mu \mathrm{M}\right)$ [32]; the proximity of the imine nitrogen may permit stabilisation of the electron-deficient oxygen radical through donation [33]. Compound $\mathbf{3 g}$, with dihydroxyl groups in ring ' $\mathbf{A}$ ' as well as ortho- $\mathrm{OH}$ in ring ' $\mathbf{B}$ ', was the most potent of the IRAs prepared [34]. Additionally, derivatives in the IRA series were more able to quench singlet oxygen than the parent resveratrol $\mathbf{3 b}$ (determined via TEMP- ${ }^{1} \mathrm{O}_{2}$ adduct formation assay). Imine resveratrol analogues have been investigated in several other independent studies and have shown similar activities and SAR trends [35]. Furthermore the methoxylated IRA 3i demonstrated antiinflammatory activity in an in vivo mouse model [36]. These data demonstrate that either olefins or imines in the stilbenoid scaffold can support antioxidant activity, and further emphasise the importance of hydroxyl groups in radical formation and stabilisation.

Extensive structural modifications to the stilbenoid scaffold are permitted whilst still retaining or even enhancing antioxidant activity, provided that the key structural features required for activity are retained. Several tetrahydropyrroyl- (THP) derivatives (4b) were analysed alongside their stilbenoids counterparts (4a) for their ability to block DNA oxidation in a thiobarbituric acid assay [37, 38]. All THP derivatives were found to be more potent antioxidants than their stilbenoid counterparts. With regards to substituent effects, 


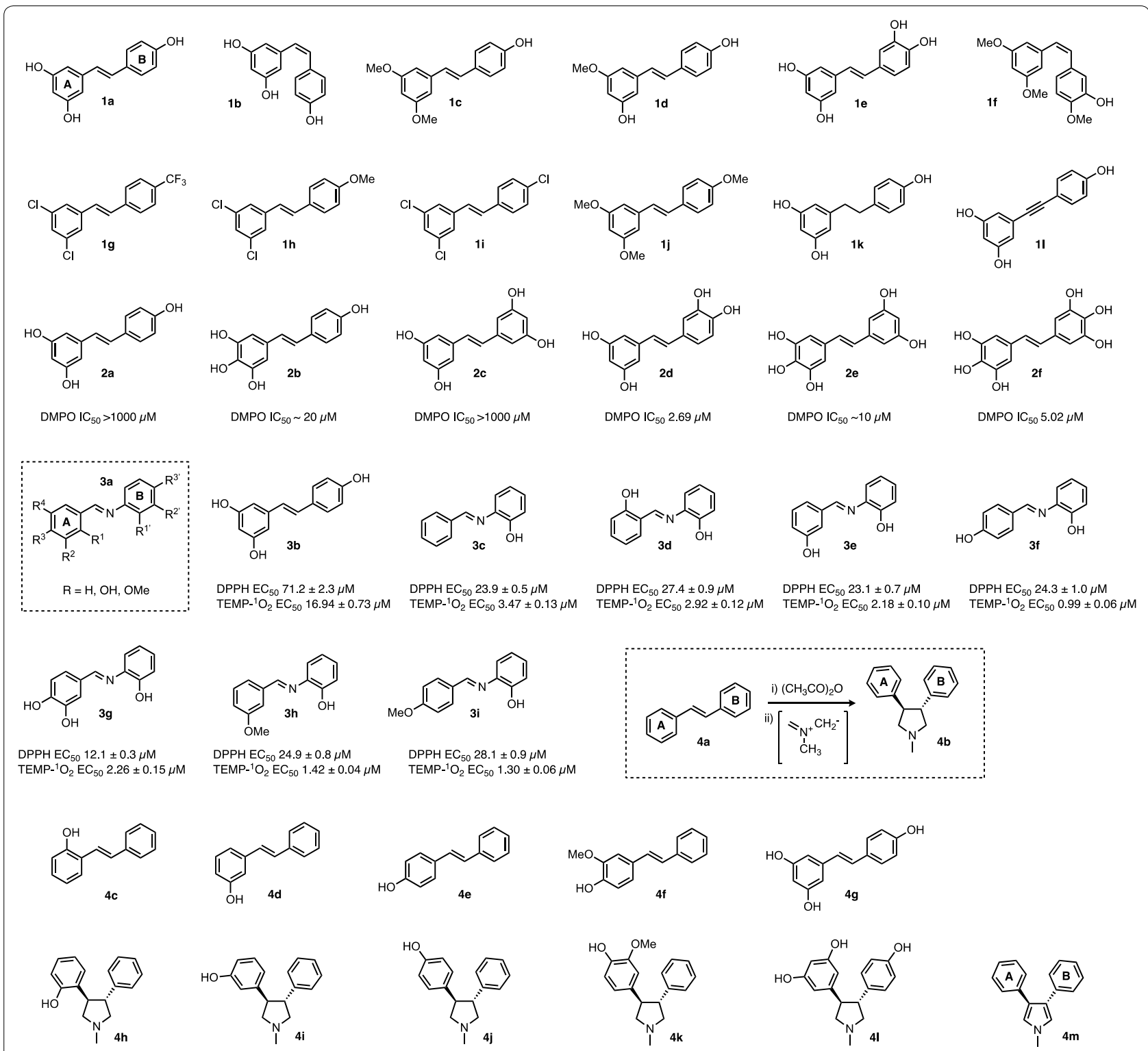

Fig. 2 Resveratrol and stilbenoid derivatives. Groups 1-4 reflect the primary research paper(s) in which the series shown was first described $(\mathbf{1} \mathbf{a}-\mathbf{1} \mathbf{l}=\operatorname{ref}[25] ; \mathbf{2} \mathbf{a}-\mathbf{2} \mathbf{f}=\operatorname{ref}[28] ; \mathbf{3} \mathbf{a}-\mathbf{3 i}=\operatorname{ref}[29] ; \mathbf{4 a}-\mathbf{4 m}=$ Refs. [34, 35]). Where given in the literature, efficacy in antioxidant/radical quenching assays is shown. (Note resveratrol was studied in all four groups and is shown as entries $\mathbf{1} \mathbf{a}, \mathbf{2} \mathbf{a}, \mathbf{3} \mathbf{b}$ and $\mathbf{4} \mathbf{g}$ for comparison). In vitro activity dataDMPO EC ${ }_{50}$ concentration giving $50 \%$ of max effect in DMPO superoxide radical scavenging assay. TEMP- ${ }^{1} \mathrm{O}_{2}$ - singlet oxygen quenching. DPPH -DPPH nitrogen radical scavenging assay. General schemes or scaffolds are enclosed in dotted lines

2-hydroxy phenyl $4 \mathrm{~h}$ was the most potent antioxidant, followed by para $\mathbf{4} \mathbf{j}$ then meta $4 \mathbf{i}$. Derivatives containing electron donating groups such as 3-methoxy $\mathbf{4 k}$ possessed stronger antioxidant capacity, potentially permitting stabilisation of the radical intermediates. The most potent antioxidant was synthesized through tetrahydropyrrollidisation of resveratrol $\mathbf{4 g}$ itself, to yield 4l, though no other tri-hydroxylated THP derivatives were studied. The function of the THP group as a chemical bystander was also investigated; it was found that oxidation of the tetrahydropyrrole to the pyrrole 4m occurs in situ, indicating that the THP group itself is stoichiometrically antioxidant, and increases activity in a mechanistic manner as opposed to just altering the stability of the phenoxy radicals. 


\section{The structural basis of direct antioxidant activity of flavonoids}

The antioxidant capabilities of flavonoids follow similar trends to the stilbenoids but are more nuanced, due to the different effects of flavonoid core scaffolds, as illustrated in Fig. 3 entries 5a-5e.

The HOMO/LUMO energies and ionisation potentials of 10 flavonoid scaffolds including (but not limited to) $5 \mathbf{a}-5 \mathbf{e}$ were analysed in a density functional theory study, in order to determine the effects of the core scaffold on the theoretical anti-oxidant capacity [39]. A hydroxyl group in the 3-position of the flavonoid scaffold and a $\mathrm{C}^{2}=\mathrm{C}^{3}$ double bond $\mathbf{5 d}$ were found to have higher ionisation potential (IP) (i.e. donate electrons more efficiently), and to stabilise the resultant flavonoid cation free radical (stabilisation energy $\Delta \mathrm{E}_{\text {iso }}$ ) than scaffolds lacking these structural features. Additionally, ether groups in the scaffold help to stabilise the cation free radical through resonance. The flavonol scaffold $\mathbf{5 d}$ possesses all of these faculties and indeed is experimentally found to be one of the most potent antioxidant structures in the flavonoid class; stepwise masking of these features serves to diminish activity.

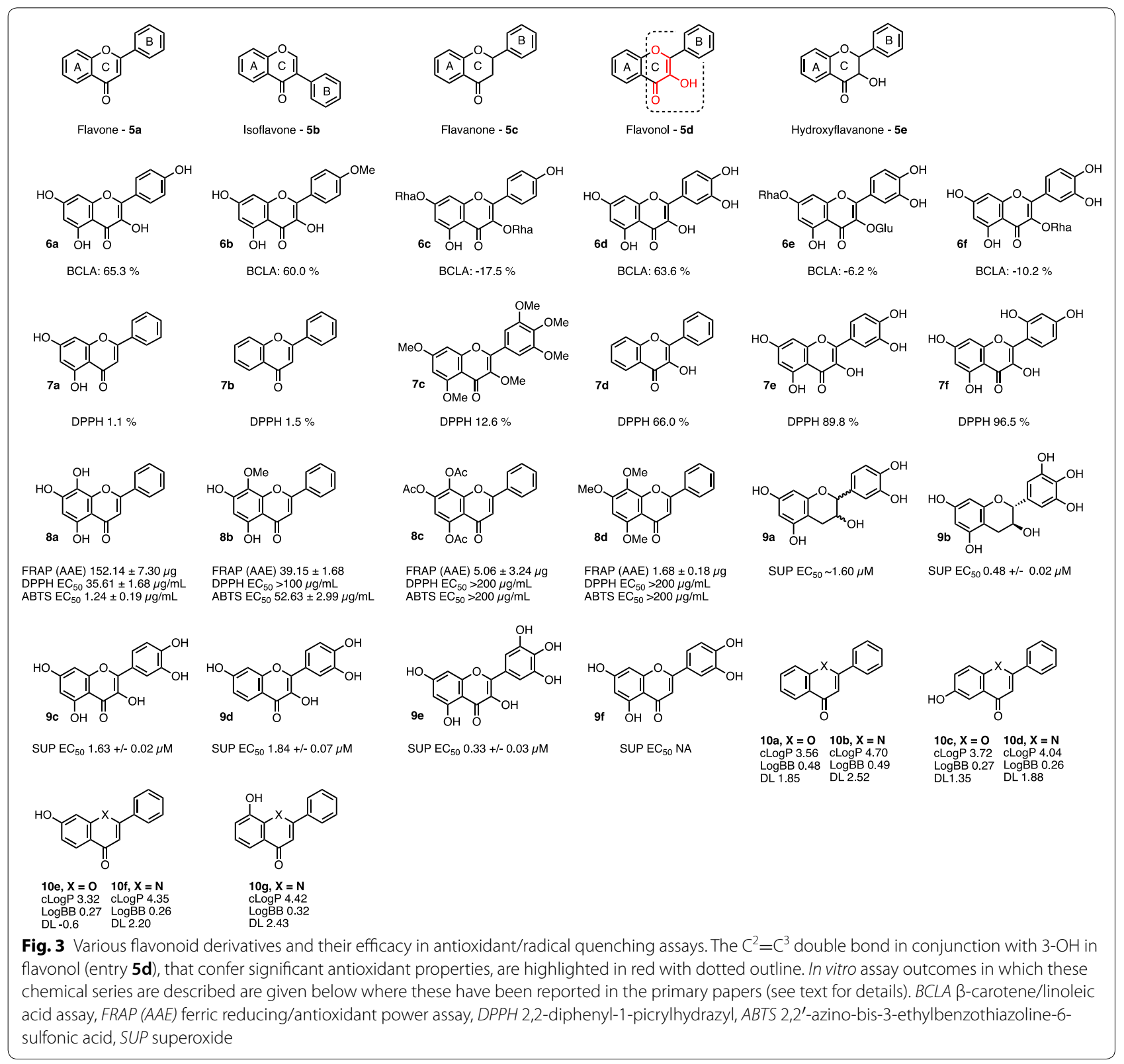


The radical scavenging and anti-oxidant capabilities of forty-one flavonoids were benchmarked against each other in DPPH and $\beta$-carotene/linoleic acid assays (BCLA-expressed as \% antioxidant activity) [40]. The scaffold, 5d, Fig. 3 was sufficient in itself to provide increased protection against oxidation in the $\beta$-carotene/ linoleic acid assay. Masking of the $\mathrm{C}^{3}-\mathrm{OH}$ in ring ' $\mathrm{C}$ ' $\mathbf{6 c}$, 6e-6f was in turn sufficient to ablate antioxidant activity. A comparison of radical scavenging in the DPPH assay (expressed as \% radical quenching) demonstrated that compounds with flavonols possessing $4^{\prime} \mathrm{OH}$ and/or ortho di-OH groups in the $\mathrm{B}$ ring were in general the most effective, and activity decreased as these structural features were removed, though there are exceptions, cf. $7 \mathbf{a}-$ $7 \mathbf{f}$. As observed in the stilbenoids, the presence of $\mathrm{OH}$ groups were required for radical scavenging. While the presence of $\mathrm{OH}$ groups in the ring ' $\mathrm{A}$ ' was not sufficient for radical scavenging (demonstrated by compounds $7 \mathbf{a}$, $\mathbf{7 d}, \mathbf{7 e}$ ), catechol functionality (ortho di-OH) in ring ' $\mathbf{A}$ ' is sufficient, thus norwogonin 8a possesses activity in multiple antioxidant assays, but it rapidly loses activity upon partial or complete masking of the hydroxyl groups, as shown in derivatives $\mathbf{8 b - 8 d}$ [41].

Catechol functional groups are sufficient to permit the scavenging of superoxide radicals $\left(\mathrm{O}_{2}^{--}\right)$in flavonols/flavanols as well as stilbenoids, as demonstrated by flavonoids $9 \mathbf{a}-9 \mathbf{e}$, which also possess the ability to scavenge superoxide radicals [42]. Additionally, comparisons can be drawn between quercetin $\mathbf{9 c}$ and the flavone luteolin 9f, which is identical to quercetin in terms of hydroxyl group distribution on the phenyl rings ' $A$ ' and ' $B$ ', yet which cannot directly scavenge $\mathrm{O}_{2}^{-\cdot}$, indicating a greater ability of the flavonol scaffold to stabilise the resultant free radicals (though luteolin does possess indirect antioxidant capacity through the inhibition of xanthine oxidase). Importantly, quercetin (in combination with kinase inhibitor dasatinib) is proving of clinical value in diseases such as idiopathic lung fibrosis and diabetic kidney disease [43, 44]. While benefit has been attributed to senolytic activity (selective killing of senescent cells), antioxidant activity of quercetin in this context may also be important, possibly by preventing further ROS-induced senescence. For both the stilbenoid and flavonoid scaffolds, there are several instances where compounds containing catechol (ortho-dihydroxyl) functionality possess more potent radical scavenging activity than would otherwise be expected from two non-contiguous hydroxyl group based on the SAR, e.g. structures $7 \mathbf{a} / \mathbf{8 a}, 9 \mathbf{a} / \mathbf{9 b}$ etc. This effect is due to neighbouring group participation in hydrogen bonding and in stabilisation of the resultant radical $[34,45]$.

SAR investigations towards optimisation of radical scavenging ability (i.e. in vitro efficacy) still present only a part of a successful medicinal chemistry campaignthe molecules must also be appropriately absorbed and delivered to their sub-cellular site of action in the body, so improvement of the ADME profile of the molecules is an important consideration in delivering effective therapeutics. In flavonols, the oxygen atom in ring ' $\mathbf{C}$ ' can be substituted for nitrogen, as with the respective quinolones $(\mathbf{1 0 a}-10 \mathrm{~g})$ [46] which retain their antioxidant capacity, but additionally displaying preferable ADME profiles. While the 7-OH 10f substitution increased activity in scavenging peroxyl radicals (ORAC), 6-OH and 8-OH quinolones 10d and $10 \mathrm{~g}$ showed increased scavenging activity towards hydroxyl radicals as well as ferrous iron reduction (TBARS and FRAP assays, respectively). Iron levels increase up to 30-fold in pro-inflammatory senescent cells (which also show high endogenous ROS levels) [47], so it will be interesting to test whether iron reduction by quinolones is biologically relevant in agerelated diseases where senescence is causative. The quinolones were generally more potent than their flavone counterparts, with the exception of 6-hydroxyflavone vs. 6-hydroxyquinolone (10d) in the ORAC assay. Based on electron spin resonance (ESR) analysis of flavonoid antioxidant activities in terms of number and position of $\mathrm{OH}$ groups, it has been suggested that rate of reaction rather than stoichiometry may be important for biological protection against ROS [48].

Polyphenols can upregulate antioxidant response via NRF2 Physiological responses to oxidative stress are co-ordinated within cells by the transcription factor NRF2 (Nuclear factor erythroid 2-related factor 2). Under normoxic conditions, NRF2 levels are low, predominantly due to binding to the negative regulator KEAP1 (Kelchlike ECH-associated protein 1) which facilitates NRF2 ubiquitination and proteasomal degradation [49, 50]. During increased oxidative stress, oxidative cysteine modification of KEAP1 alters its conformation, resulting in diminished binding to NRF2 [51]. NRF2, no longer subject to degradation, translocates to the nucleus where it binds to the antioxidant response element (ARE) upstream of cytoprotective genes, e.g. $\mathrm{NAD}(\mathrm{P}) \mathrm{H}$ quinone oxidoreductase 1 (NQO1), glutathione S-transferase (GST), and glutathione reductase [52, 53], inducing their expression. These factors act to lower ROS and oxidative stress, while simultaneously reducing the cysteines in KEAP1 and subsequently re-establishing a baseline equilibrium of NRF2 activity. Activation of NRF2 signalling, and concomitant amelioration of age-related phenotypes by polyphenols is well documented in vitro and in vivo, and the scaffolds serve as valuable starting points for SAR investigations [54-59]. The stilbenoids resveratrol and pterostilbene specifically have both been demonstrated 
to upregulate NRF2 activity [55, 60]. A computational study demonstrated that pterostilbene can form stabilising interactions with basic amino acids in the kelch domain of KEAP1 and thus interferes with the breakdown/negative regulation of NRF2 [60].

\section{NRF2-mediated upregulation of proteasome activity by polyphenols}

As well co-ordinating the antioxidant response, activation of NRF2 has been demonstrated to increase proteasomal activity, allowing cells to control protein levels by regulated degradation; while the proteasome degrades proteins tagged with ubiquitin, larger scale protein (and organelle) regulation within cells is mediated by autophagy. The importance of proteostasis is highlighted by the finding of downregulation of autophagy and proteasomal activity in replicatively senescent cells in vitro [61], while inhibition of the proteasome in replicating cells to levels commensurate with that seen in senescence is sufficient to induce premature cellular senescence [62, 63]. Activation of NRF2 using small molecules such as $3 \mathrm{H}$-1,2-dithiole-3-thione (D3T, 11) and $18 \alpha$-Glycyrrhetinic acid $(18 \alpha-G A, 12)$ increases the expression and activity of the proteasome in a NRF2dependant manner $[64,65]$. Activation of NRF2 by $18 \alpha-$ GA confers resistance against oxidative stress, extends the maximal replicative capacity of human lung HFL-1 fibroblasts, and delays the onset of several phenotypes of cellular senescence [65]. Activation of SKN-1 (worm orthologue of human NRF2) was also demonstrated to upregulate proteasome activity and extend life span in C. elegans [66]. Many compounds that upregulate the antioxidant response in vivo contain thiol reactive functionality, for example $\alpha, \beta$-unsaturated carbonyl groups, that can undergo conjugate addition reactions to covalently bind to their targets. Examples include chalcones and various flavonoids [67, 68]. Excitingly, the flavonoid 4,4'-dimethoxychalcone (DMC) has recently been reported to have anti-ageing effects across species, potentially through induction of autophagy [69].

NRF2 can also be modulated through imine resveratrol analogues with varied substitution patterns of hydroxylation and methoxylation [70]. Inclusion of an ortho- $\mathrm{OH}$ group in the ' $\mathbf{B}$ ' ring was found to robustly increase the potency in terms of luciferase expression in an AREluciferase reporter assay across several analogues modified across all positions in ring 'A', c.f. 13b-13f, but this effect could not be recapitulated by the same ortho-OH substitution in the ' $\mathbf{A}$ ' ring. Ortho-OH substitution in the ' $B$ ' ring also increased direct radical scavenging capacity. By combining structural features from promising analogues, two second round derivatives, $13 \mathrm{~g}$ and $\mathbf{1 3 h}$, were designed which proved to be the most potent derivatives synthesized, increasing ARE-luciferase levels by approximately 10 - and 12 -fold over control at $15 \mu \mathrm{M}$ respectively (c.f. resveratrol $\mathbf{1 3 i}$ leads to a threefold induction at $15 \mu \mathrm{M})$. The mechanism of action is proposed to be a covalent inhibition of KEAP1 through nucleophilic addition of a cysteine moiety from KEAP into the electrophilic imine. This interaction could be potentially stabilised through interaction with the $\mathrm{OH}$ increasing the reactivity of the incoming thiol, which is illustrated in entry 13j. The fact that ortho-hydroxyl groups adjacent to electrophilic sites have been shown elsewhere [71, 72] to increase the rate of reactivity with sulfhydryl moieties supports this hypothesis. IRAs represent a promising series since they demonstrate both direct and indirect radical scavenging capacity and are active in vivo [36].

The structural requirements for upregulation of AREdriven quinone reductase activity in vitro are highlighted by a comparison between eight flavonoids (Fig. 4, 14a-h). The $\alpha, \beta$-unsaturated ketone in the flavone/flavonol scaffold appears to be required for reactivity, since disruption of this structural feature completely supresses the activity of these compounds. Entries 14a-14e possess $\alpha$, $\beta$-unsaturated carbonyls with varied distribution of $\mathrm{OH}$ groups on rings $\mathbf{A}-\mathbf{C}$ and retain activity with micromolar potency. Disruption of the $\alpha, \beta$-unsaturated ketone through removing the double bond, ketone, or both (i.e. compounds 14f-14h) results in loss of activity in terms of NRF2 activation [68].

Measuring ARE-dependent NQO1 expression as a marker for NRF2 transcriptional activity [73], Fahey and colleagues conducted an assessment of a thirty-seven member flavonoid library [74]. Within this set, comparative analysis of the scaffold is possible, since there were several matched molecular pairs, wherein the compounds were identical apart from disruption of the $\alpha, \beta$-unsaturated ketone functionality. For example, flavone 15a was found to be approximately four-fold more potent in supporting NRF2-dependent transcription than its flavanone derivative 15b. It is of note that of eight flavanones tested, only one possessed comparable potency to its flavone analogue, indicating the importance of intact $\alpha, \beta$-unsaturated ketone functionality. 5-methoxy substitution of ring 'A', 15d-15g, was associated with potent induction of NQO1, without a concomitant increase in toxicity as assessed in three independent cell lines. The authors note that the differences in activity did not appear to be related to cellular uptake kinetics [74].

The ability to induce NRF2 (and hence indirectly induce an antioxidant response) was removed by altering the flavonol scaffold to the analogous quinolines, hence removing the $\alpha, \beta$-unsaturated functionality while maintaining a planar ring system. In a multitarget medicinal chemistry campaign of the flavonol 


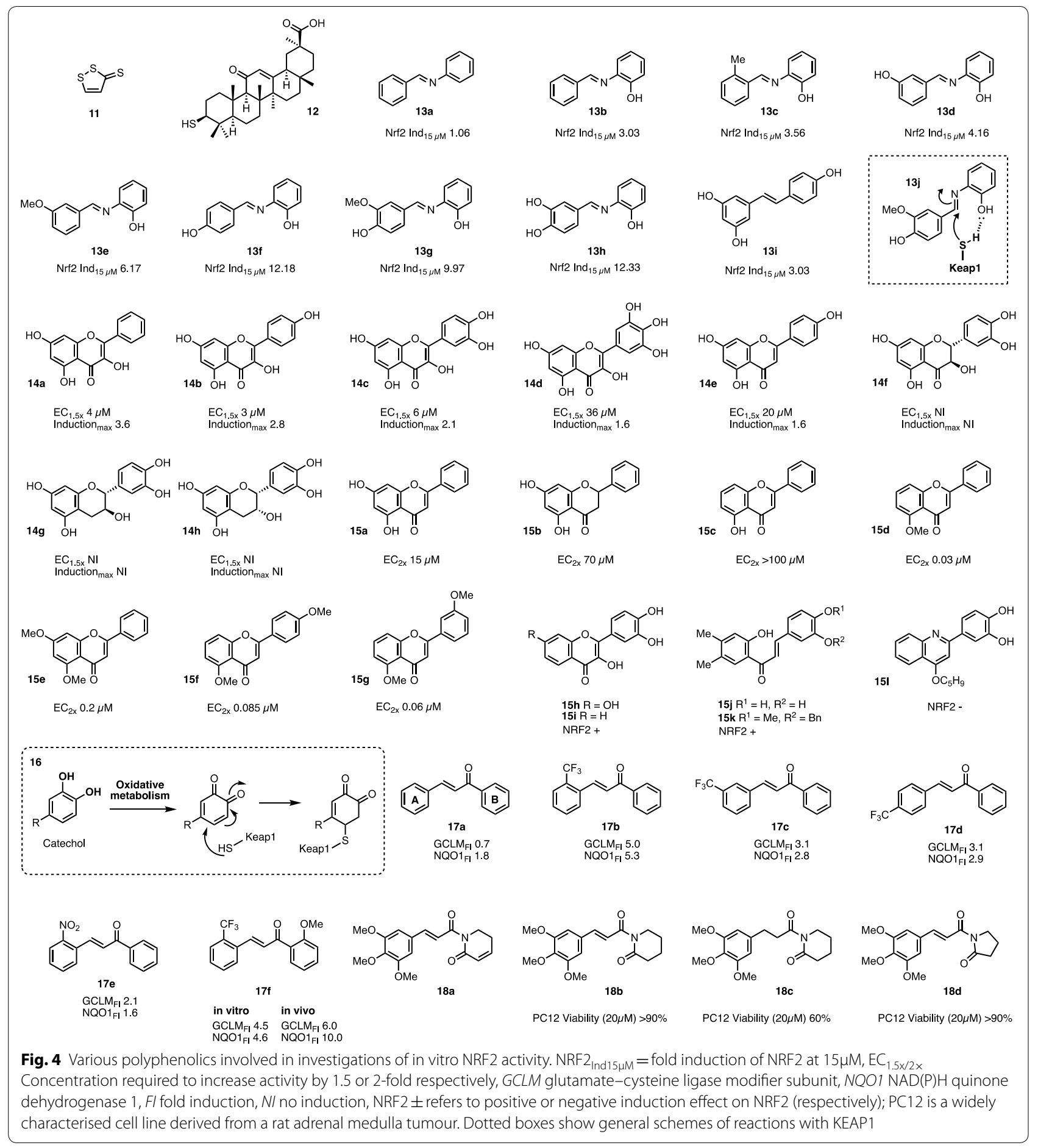

scaffold, several compounds were found to maintain GSH levels following the addition of glutamate [75]. Induction of NRF2 was assessed to determine whether these activities correlated over the series; the parent compound fisetin (entry 15h) was found to induce NRF2 activity, as well as flavonol 15i and chalcone derivatives 15j, 15k. However, activity is lost upon replacement to the quinoline scaffold (e.g. 15l), or addition of a basic nitrogen group to ring 'B', which may target the compound to acidic sub-cellular compartments such as the lysosome, thus sequestering it away from its site of action [76]. 
Several flavonoid derivatives can still result in inhibition of KEAP1 despite not possessing a Michael acceptor per se. Catechol groups can undergo oxidation under physiological conditions to produce quinones, as depicted in entry 16. This can be achieved over several steps through various mechanisms, such as oxidation with ROS or redox metalloenzymes, if the cellular environment permits it. The stable quinones can then react with thiols/thiolates on KEAP1, driving an increase in NRF2 activity; alternatively they can be sequestered through glutathione conjugation, which is in turn regenerated [77, 78]. Compounds of this class can increase NRF2 activity in vivo [79]. Furthermore, while some structures do not contain, nor have the propensity to form, $\alpha, \beta$-unsaturated carbonyls, ketones themselves (for example in flavanones) are electrophilic and could potentially react with KEAP1 or other sensor-type cysteine proteins forming the respective hemithioacetals; additionally, compounds may also act upon the NRF2/KEAP1 axis in a non-covalent manner [80-85].

NRF2 can similarly be modified by other $\alpha$, $\beta$-unsaturated ketone-containing natural products such as chalcones, from which flavonoids are structurally derived [86]. Notably these compounds exhibit activity while not containing any hydroxyl groups. A library of fifty-nine chalcones (scaffold of entry 17a) were synthesized and screened (at $100 \mu \mathrm{M}$ ) for their ability to modulate NRF2 signalling, as determined by measuring the fold change in expression of ARE-driven genes GCLM and NQO1 [87]. A large array of methoxy-substituted derivatives at ring 'A' were analysed in concert with either an ortho, meta, or para- $\mathrm{CF}_{3}$ substitution at ring 'B' (17b-17d). Ortho- $\mathrm{CF}_{3}(\mathbf{1 7 b})$ reliably improved potency of the compounds in terms of fold induction, as compared with meta and para, potentially indicating that a negative inductive effect increases the reactivity of the $\alpha, \beta$-unsaturated ketone. Attempts to recapitulate this by substitution of the $\mathrm{CF}_{3}$ group for $\mathrm{NO}_{2}(\mathbf{1 7 d})$ rendered the compounds cytotoxic [87]. The pattern of methoxy substitution on the phenyl ring did not show a consistent or discernible SAR profile: the most potent induction of NRF2 in vitro was seen with the unsubstituted ortho$\mathrm{CF}_{3}$ derivative $\mathbf{1 7} \mathbf{b}$, but disubstituted $\mathbf{1 7 f}$ emerged as the most potent of the series when the compounds were examined in vivo in mouse small intestine following gavage (6- and 10-fold upregulation for GCLM and NQO1 respectively compared to vehicle control) [87].

Piperlongumine (PL, 18a) is a natural product chalcone-derivative with demonstrated cytoprotective effects against $\mathrm{H}_{2} \mathrm{O}_{2}$ or 6-OHDA-induced oxidative stress; the protective mechanism is probably mediated through NRF2 signalling [88] As with the parent chalcone scaffold, retention of the exocyclic trans double bond was found to be necessary for cytoprotection against oxidative stress, as demonstrated by pairwise analysis of compounds $18 \mathrm{~b}$ and 18c. The olefin present in the lactam ring in parent PL 18a was not required for potent activation of the antioxidant response. Structures $\mathbf{1 8 b}$ and $\mathbf{1 8 d}$ were identified as the most potent derivatives lacking innate cytotoxicity and were shown to induce the nuclear accumulation of NRF2, with a concomitant upregulation of the antioxidant response. Knockdown of NRF2 ablated the cytoprotective effect of these compounds, demonstrating their action through the NRF2 axis [88].

\section{SAR summary}

The ability of both stilbenoids and flavonoids to directly scavenge free radical species is dependent on the presence of a labile hydrogen within the polyphenol, usually from $\mathrm{OH}$, to donate to ROS, and also on the resultant stability of the polyphenol radical once formed. Masking of critical $\mathrm{OH}$ groups required for reactivity with ROS ablates potency. Similarly, antioxidant activity of polyphenols is also decreased by destabilisation of the resultant radical through removal of the stilbenoid $\pi$-bond (olefin or imine), removing scaffold-defining features of flavonols, or substitution on phenyl groups with substituents that destabilise the radical.

With regards to NRF2 activation, $\alpha, \beta$-unsaturated carbonyl groups appear to be necessary for polyphenols to act as indirect antioxidants though activation of NRF2. Soft electrophiles are preferred since the mechanism is through reaction with KEAP1 cysteine residues ( $\mathrm{R}-\mathrm{SH}$ being a soft nucleophile). To our knowledge, it has not been conclusively demonstrated whether the less reactive $\alpha, \beta$-unsaturated carbonyl groups that are present in the scaffold react directly with KEAP1, or whether said scaffold serves to stabilise the formation of more reactive quinone species in the neighbouring phenol ring, which would subsequently react with KEAP1. Regardless of mechanism, the strong dependence on the presence of the $\alpha, \beta$-unsaturated carbonyls for efficacy is notable. Flavonoids containing imines and ketones retain some potency. Modifications enhancing electrophilic reactivity appear to also enhance in vitro potency for NRF2 activation. Sensitivity of various structural features towards modification are described in Fig. 5.

\section{Conclusions}

Age-related diseases (ARDs) are complex, systemsdependent phenomena; drugs that act on individual protein targets are therefore unlikely to be effective in disease modification. By contrast, compounds that possess beneficial polypharmacology, i.e. target multiple therapeutic pathways simultaneously, will have a higher likelihood of robustly modulating ARDs. Polyphenolics such as 


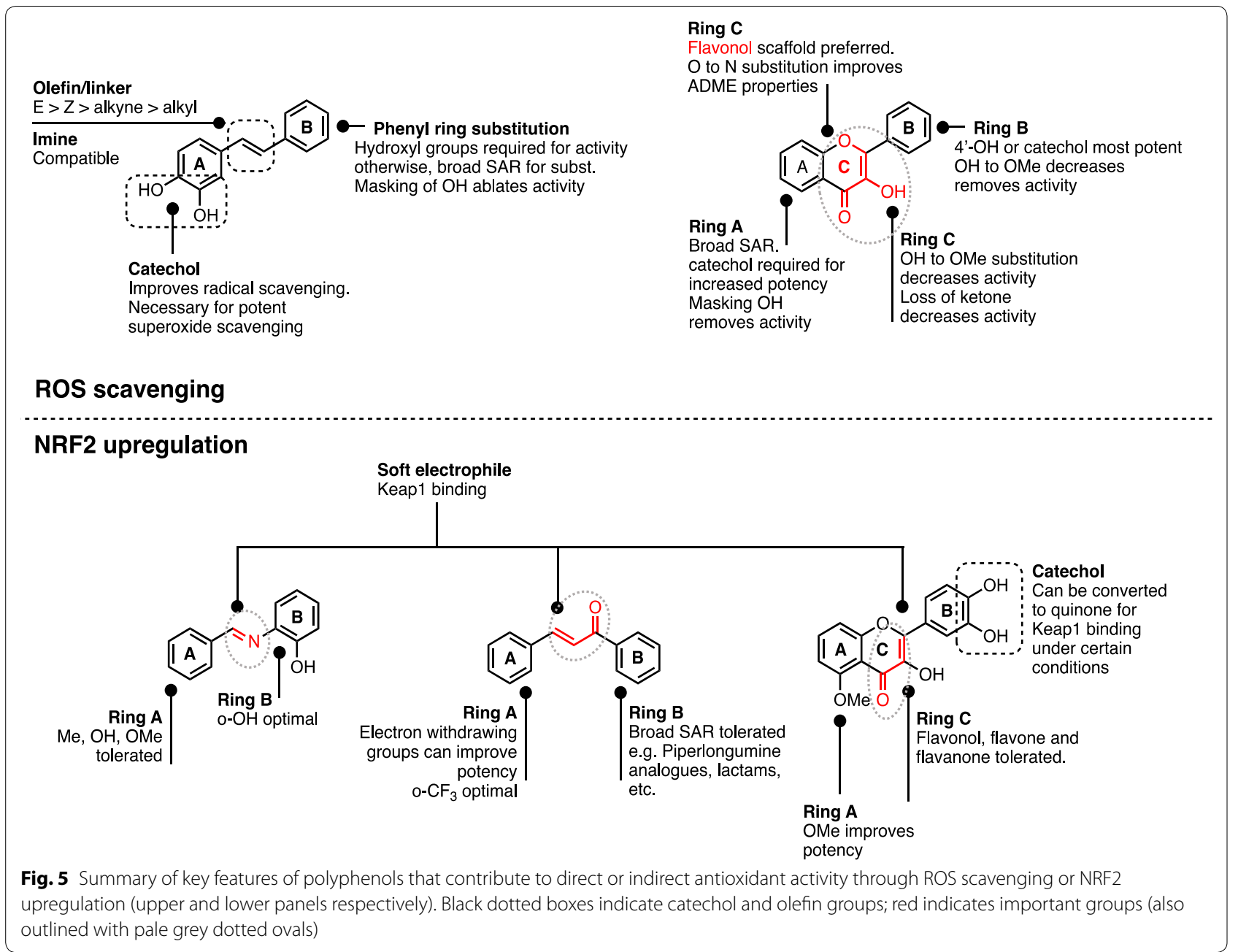

stilbenoids, flavonoids and chalcones are polypharmacologically active in several ARD-relevant biological processes, particularly oxidative stress, inflammation and cellular senescence. The pharmacophores for direct and indirect modulation of oxidative stress, through scavenging of ROS or upregulation of NRF2 activity respectively, are overlapping in some areas and discrete in others, but both direct and indirect activities are likely to be important in the overall molecular mechanisms of these compounds in vivo. Additionally, there are other contributing mechanisms by which specific sub-classes of polyphenolics may affect ROS homeostasis not discussed here, such as inhibition of xanthine oxidase [42]. This complexity mirrors that of the multiple interacting biochemical pathways involved in generating the complex phenotype of biological ageing. In drug design campaigns for antiageing therapeutics, we therefore caution against optimising for efficacy in one particular facet of biochemical activity (e.g. in vitro quenching of ROS) without reflecting the complexity of the biological system [89] such as that represented by cell and organismal ageing. Moreover, while transformed cancer cell lines are widely used in drug screening (presumably because of ease of culture and immortality), they are far from ideal as a platform for testing drugs for amelioration of ageing phenotypes; such immortal cells do not age and their biochemical signalling pathways can be highly abnormal. Instead, therefore, we suggest that anti-ageing drug development requires testing in a number of parallel in vitro biochemical assays combined with full phenotypic assays using cell and/ or whole organism models directly relevant to ageing, for example replicatively aged primary human cells and short-lived invertebrate organisms with readily scorable ageing outcomes. Such screening programmes are likely to highlight agents with beneficial polypharmacological properties that may prove to have therapeutic benefit in vivo. Polyphenolic natural products therefore represent strong starting points for medicinal chemistry optimisation campaigns in anti-ageing therapeutics. 


\begin{abstract}
Abbreviations
6-OHDA: 6-Hydroxydopamine; 18a-GA: 18a-Glycyrrhetinic acid; ABTS: 2,2'-Azino-bis-3-ethylbenzothiazoline-6-Sulfonic acid; ARD: Age-related disease; ARE: Antioxidant response element; ATP: Adenosine triphosphate; BCLA: B-CAROTENE/linoleic acid assay; D3T: 3H-1,2-Dithiole-3-thione; DMPO: 5,5-Dimethyl-1-pyrroline-N-oxide; DNA: Deoxyribonucleic acid; DPPH: 2,2-Diphenyl-1-picrylhydrazyl; ESR: Electron spin resonance; Fl: Fold induction; FRAP: Ferric reducing/antioxidant power; FRAP (AAE): Ferric reducing/ antioxidant power (ascorbic acid equivalents); GCLM: Glutamate-cysteine ligase modifier subunit; GSH: Glutathione; GST: Glutathione S-transferase; IRA: Imine resveratrol analogues; KEAP1: Kelch-like ECH-associated protein 1; NI: No induction; NRF2: Nuclear factor erythroid 2-related factor 2; ORAC : Oxygen radical absorbance capacity; ROS: Reactive oxygen species; SAR: Structure-activity relationship; SKN-1: Nematode worm orthologue of human NRF2; SUP: Superoxide; TBARS: Thiobarbituric acid reactive substances; TEMP: (2,2,6,6-Tetramethylpiperidin-1-yl)-; THP: Tetrahydropyrroyl.
\end{abstract}

\section{Acknowledgements}

Not applicable.

\section{Authors' contributions}

AR conducted the review of the literature; AR and LSC co-wrote the manuscript. Both authors read and approved the final manuscript.

\section{Authors' information}

AR is a Research Fellow in the Department of Biochemistry with a strong research interest at the interface of chemical biology and ageing, as well as developing translational methods to better understand underlying principles of drug action. AR completed his PhD at the National Centre for Advancing Translational Sciences (NCATS/NIH) with a focus on phenotype-driven medicinal chemistry. LSC is an Associate Professor of Biochemistry studying the biological basis of ageing, with the aim of developing effective therapies to ameliorate age-related disease based on treating underlying biological ageing processes. Both AR and LSC are trustees of the British Society for Research on Ageing. LSC is the George Moody Fellow in Biochemistry at Oriel College, Oxford, a member of the Biochemical Society's Clinical and Translational theme panel, and a scientific advisor to the All Party Parliamentary Group for Longevity.

\section{Funding}

AR is funded by Elysium Health through a personal Oxford-Elysium Fellowship for drug discovery for age-related diseases. The work in the lab of LSC is also funded by Diabetes UK through a BIRAX award (ref 19BX18LCRD), UK SPINE/ Research England, and through philanthropic donations. She is grateful to the BBSRC for grant BB/M006727/1. The funders had no involvement in the design of the study, in collection, analysis and interpretation of data, or writing of the manuscript.

\section{Availability of data and materials}

All data generated or analysed during this study are included in this published article.

\section{Competing interests}

AR is funded by Elysium Health, a manufacturer of dietary supplements. LSC and AR have previously received funding from Access Business Group for studies on anti-inflammatory properties of natural products.

\section{Received: 5 March 2020 Accepted: 10 July 2020}

Published online: 10 August 2020

\section{References}

1. Kingston A, Robinson L, Booth H, Knapp M, Jagger C, for the MODEM project (2018) Projections of multi-morbidity in the older population in England to 2035: estimates from the population ageing and care simulation (PACSim) model. Age Ageing 47(3):374-380

2. Harman D (1956) Aging: a theory based on free radical and radiation chemistry. J Gerontol 11(3):298-300

3. Liguori I, Russo G, Curcio F, Bulli G, Aran L, Della-Morte D et al (2018) Oxidative stress, aging, and diseases. Clin Interv Aging 13:757-772
4. Gladyshev VN (2014) The free radical theory of aging is dead. Long live the damage theory! Antioxid Redox Signal 20(4):727-731

5. Hernandez-Segura A, Nehme J, Demaria M (2018) Hallmarks of cellular senescence. Trends Cell Biol 28(6):436-453

6. López-Otín C, Blasco MA, Partridge L, Serrano M, Kroemer G (2013) The hallmarks of aging. Cell 153(6):1194-1217

7. Ahuja I, Kissen R, Bones AM (2012) Phytoalexins in defense against pathogens. Trends Plant Sci 17(2):73-90

8. Mathers JC (2015) Impact of nutrition on the ageing process. $\mathrm{Br} J$ Nutr 113(S1):S18-S22

9. Baur JA, Sinclair DA (2006) Therapeutic potential of resveratrol: the in vivo evidence. Nat Rev Drug Discov 5(6):493-506

10. Panche AN, Diwan AD, Chandra SR (2016) Flavonoids: an overview. J Nutr Sci. 5:e47

11. Zhuang C, Zhang W, Sheng C, Zhang W, Xing C, Miao Z (2017) Chalcone: a privileged structure in medicinal chemistry. Chem Rev 117(12):7762-7810

12. Tsao R (2010) Chemistry and biochemistry of dietary polyphenols. Nutrients. 2(12):1231-1246

13. Barbosa MC, Grosso RA, Fader CM (2019) Hallmarks of aging: an autophagic perspective. Front Endocrinol 9:790

14. Höhn A, Weber D, Jung T, Ott C, Hugo M, Kochlik B et al (2017) Happily (n) ever after: aging in the context of oxidative stress, proteostasis loss and cellular senescence. Redox Biol 11:482-501

15. Losada-Barreiro S, Bravo-Díaz C (2017) Free radicals and polyphenols: the redox chemistry of neurodegenerative diseases. Eur J Med Chem 133:379-402

16. Reczek CR, Chandel NS (2015) ROS-dependent signal transduction. Curr Opin Cell Biol 33:8-13

17. Cai Z, Yan L-J (2013) Protein oxidative modifications: beneficial roles in disease and health. J Biochem Pharmacol Res. 1(1):15-26

18. Bertram C, Hass R (2008) Cellular responses to reactive oxygen speciesinduced DNA damage and aging. Biol Chem 389(3):211-220

19. McHugh D, Gil J (2018) Senescence and aging: causes, consequences, and therapeutic avenues. J Cell Biol 217(1):65-77

20. te Poele RH, Okorokov AL, Jardine L, Cummings J, Joel SP (2002) DNA Damage is Able to Induce Senescence in Tumor Cells in vitro and in vivo. Cancer Res 62(6):1876

21. Hill S, Lamberson CR, Xu L, To R, Tsui HS, Shmanai W et al (2012) Small amounts of isotope-reinforced polyunsaturated fatty acids suppress lipid autoxidation. Free Radic Biol Med. 53(4):893-906

22. Hill S, Hirano K, Shmanai VV, Marbois BN, Vidovic D, Bekish AV et al (2011) Isotope-reinforced polyunsaturated fatty acids protect yeast cells from oxidative stress. Free Radic Biol Med. 50(1):130-138

23. Hatami A, Zhu C, Relano-Gines A, Elias C, Galstyan A, Jun M et al (2018) Deuterium-reinforced linoleic acid lowers lipid peroxidation and mitigates cognitive impairment in the Q140 knock in mouse model of Huntington's disease. FEBS J 285(16):3002-3012

24. Beaudoin-Chabot C, Wang L, Smarun AV, Vidović D, Shchepinov MS, Thibault G (2019) Deuterated polyunsaturated fatty acids reduce oxidative stress and extend the lifespan of C. elegans. Front Physiol 10:641

25. Nóbrega-Pereira S, Fernandez-Marcos PJ, Brioche T, Gomez-Cabrera MC, Salvador-Pascual A, Flores JM et al (2016) G6PD protects from oxidative damage and improves healthspan in mice. Nat Commun 7(1):10894

26. Zhou L, Fu X, Luo Y, Du F, Wang H, Xing S et al (2017) 2-SeCD treatment extends lifespan, improves healthspan and enhances resistance to stress in Caenorhabditis elegans. RSC Adv 7(76):48245-48252

27. Storniolo CE, Moreno JJ (2019) Resveratrol analogs with antioxidant activity inhibit intestinal epithelial cancer Caco-2 cell growth by modulating arachidonic acid cascade. J Agric Food Chem 67(3):819-828

28. Brand-Williams W, Cuvelier ME, Berset C (1995) Use of a free radical method to evaluate antioxidant activity. LWT 28(1):25-30

29. Stivala LA, Savio M, Carafoli F, Perucca P, Bianchi L, Maga G et al (2001) Specific structural determinants are responsible for the antioxidant activity and the cell cycle effects of resveratrol. J Biol Chem 276(25):22586-22594

30. Antus C, Radnai B, Dombovari P, Fonai F, Avar P, Matyus P et al (2015) Antiinflammatory effects of a triple-bond resveratrol analog: structure and function relationship. Eur J Pharmacol 748:61-67

31. Murias M, Jäger W, Handler N, Erker T, Horvath Z, Szekeres T et al (2005) Antioxidant, prooxidant and cytotoxic activity of hydroxylated 
resveratrol analogues: structure-activity relationship. Biochem Pharmacol 69(6):903-912

32. Lu J, Li C, Chai YF, Yang DY, Sun CR (2012) The antioxidant effect of imine resveratrol analogues. Bioorg Med Chem Lett 22(17):5744-5747

33. Liu ZQ (2007) How many free radicals can be trapped by (Hydroxylphenylimino)methylphenol in the free-radical-induced peroxidation of triolein in micelles? QSAR Comb Sci 26(4):488-495

34. Wright JS, Johnson ER, DiLabio GA (2001) Predicting the activity of phenolic antioxidants: theoretical method, analysis of substituent effects, and application to major families of antioxidants. J Am Chem Soc 123(6):1173-1183

35. Kotora P, Sersen F, Filo J, Loos D, Gregan J, Gregan F (2016) The scavenging of DPPH, Galvinoxyl and ABTS radicals by imine analogs of resveratrol. Molecules 21(1):E127

36. Zimmermann-Franco DC, Esteves B, Lacerda LM, Souza IO, Santos JA, Pinto NCC et al (2018) In vitro and in vivo anti-inflammatory properties of imine resveratrol analogues. Bioorg Med Chem 26(17):4898-4906

37. Bao LL, Liu ZQ (2016) Tetrahydropyrrolization of resveratrol and other stilbenes improves inhibitory effects on DNA oxidation. ChemMedChem 11(15):1617-1625

38. Ghani MA, Barril C, Bedgood DR Jr, Prenzler PD (2017) Measurement of antioxidant activity with the thiobarbituric acid reactive substances assay. Food Chem 230:195-207

39. Mendes APS, Borges RS, Neto AMJC, de Macedo LGM, da Silva ABF (2012) The basic antioxidant structure for flavonoid derivatives. J Mol Model 18(9):4073-4080

40. Burda S, Oleszek W (2001) Antioxidant and antiradical activities of flavonoids. J Agric Food Chem 49(6):2774-2779

41. Sarian MN, Ahmed QU, Mat So'ad SZ, Alhassan AM, Murugesu S, Perumal $\checkmark$ et al (2017) Antioxidant and antidiabetic effects of flavonoids: a structure-activity relationship based study. Biomed Res Int 2017:8386065

42. Cos P, Ying L, Calomme M, Hu JP, Cimanga K, Van Poel B et al (1998) Structure-activity relationship and classification of flavonoids as inhibitors of xanthine oxidase and superoxide scavengers. J Nat Prod 61(1):71-76

43. Justice JN, Nambiar AM, Tchkonia T, LeBrasseur NK, Pascual R, Hashmi SK et al (2019) Senolytics in idiopathic pulmonary fibrosis: results from a firstin-human, open-label, pilot study. EBioMedicine. 40:554-563

44. Hickson LJ, Langhi Prata LGP, Bobart SA, Evans TK, Giorgadze N, Hashmi SK et al (2019) Senolytics decrease senescent cells in humans: preliminary report from a clinical trial of Dasatinib plus Quercetin in individuals with diabetic kidney disease. EBioMedicine. 47:446-456

45. Shang Y-J, Qian Y-P, Liu X-D, Dai F, Shang X-L, Jia W-Q et al (2009) Radicalscavenging activity and mechanism of resveratrol-oriented analogues: influence of the solvent, radical, and substitution. J Organic Chem 74(14):5025-5031

46. Greeff J, Joubert J, Malan SF, van Dyk S (2012) Antioxidant properties of 4-quinolones and structurally related flavones. Bioorg Med Chem 20(2):809-818

47. Masaldan S, Clatworthy SAS, Gamell C, Meggyesy PM, Rigopoulos AT, Haupt $S$ et al (2018) Iron accumulation in senescent cells is coupled with impaired ferritinophagy and inhibition of ferroptosis. Redox Biol. 14:100-115

48. McPhail DB, Hartley RC, Gardner PT, Duthie GG (2003) Kinetic and stoichiometric assessment of the antioxidant activity of flavonoids by electron spin resonance spectroscopy. J Agric Food Chem 51 (6):1684-1690

49. Itoh K, Wakabayashi N, Katoh Y, Ishii T, Igarashi K, Engel JD et al (1999) Keap 1 represses nuclear activation of antioxidant responsive elements by Nrf2 through binding to the amino-terminal Neh2 domain. Genes Dev 13(1):76-86

50. Cullinan SB, Gordan JD, Jin J, Harper JW, Diehl JA (2004) The Keap1-BTB protein is an adaptor that bridges Nrf2 to a Cul3-based E3 ligase: oxidative stress sensing by a Cul3-Keap1 ligase. Mol Cell Biol 24(19):8477-8486

51. Dinkova-Kostova AT, Holtzclaw WD, Cole RN, Itoh K, Wakabayashi N, Katoh $Y$ et al (2002) Direct evidence that sulfhydryl groups of Keap1 are the sensors regulating induction of phase 2 enzymes that protect against carcinogens and oxidants. Proc Natl Acad Sci U S A. 99(18):11908-11913

52. Nguyen T, Nioi P, Pickett CB (2009) The Nrf2-antioxidant response element signaling pathway and its activation by oxidative stress. J Biol Chem 284(20):13291-13295

53. Chorley BN, Campbell MR, Wang X, Karaca M, Sambandan D, Bangura F et al (2012) Identification of novel NRF2-regulated genes by
ChIP-Seq: influence on retinoid $X$ receptor alpha. Nucleic Acids Res 40(15):7416-7429

54. Ungvari Z, Bagi Z, Feher A, Recchia FA, Sonntag WE, Pearson K et al (2010) Resveratrol confers endothelial protection via activation of the antioxidant transcription factor Nrf2. Am J Physiol Heart Circ Physiol 299(1):H18-H24

55. Kim EN, Lim JH, Kim MY, Ban TH, Jang IA, Yoon HE et al (2018) Resveratrol, an Nrf2 activator, ameliorates aging-related progressive renal injury. Aging (Albany NY). 10(1):83-99

56. Li XN, Ma LY, Ji H, Qin YH, Jin SS, Xu LX (2018) Resveratrol protects against oxidative stress by activating the Keap-1/Nrf2 antioxidant defense system in obese-asthmatic rats. Exp Ther Med. 16(6):4339-4348

57. Magesh S, Chen Y, Hu L (2012) Small molecule modulators of Keap1-Nrf2ARE pathway as potential preventive and therapeutic agents. Med Res Rev 32(4):687-726

58. Singh B, Shoulson R, Chatterjee A, Ronghe A, Bhat NK, Dim DC et al (2014) Resveratrol inhibits estrogen-induced breast carcinogenesis through induction of NRF2-mediated protective pathways. Carcinogenesis 35(8): 1872-1880

59. Sirerol JA, Feddi F, Mena S, Rodriguez ML, Sirera P, Aupi M et al (2015) Topical treatment with pterostilbene, a natural phytoalexin, effectively protects hairless mice against UVB radiation-induced skin damage and carcinogenesis. Free Radic Biol Med. 85:1-11

60. Bhakkiyalakshmi E, Dineshkumar K, Karthik S, Sireesh D, Hopper W, Paulmurugan R et al (2016) Pterostilbene-mediated Nrf2 activation: mechanistic insights on Keap1:Nrf2 interface. Bioorg Med Chem 24(16):3378-3386

61. Chondrogianni N, Stratford FLL, Trougakos IP, Friguet B, Rivett AJ, Gonos ES (2003) Central role of the proteasome in senescence and survival of human fibroblasts: induction of a senescence-like phenotype upon its inhibition and resistance to stress upon its activation. J Biol Chem 278(30):28026-28037

62. Chondrogianni N, Trougakos IP, Kletsas D, Chen QM, Gonos ES (2008) Partial proteasome inhibition in human fibroblasts triggers accelerated $\mathrm{M} 1$ senescence or M2 crisis depending on $\mathrm{p} 53$ and Rb status. Aging Cell 7(5):717-732

63. Chondrogianni N, Gonos ES (2004) Proteasome inhibition induces a senescence-like phenotype in primary human fibroblasts cultures. Biogerontology 5(1):55-61

64. Kwak M-K, Wakabayashi N, Greenlaw JL, Yamamoto M, Kensler TW (2003) Antioxidants enhance mammalian proteasome expression through the Keap1-Nrf2 signaling pathway. Mol Cell Biol 23(23):8786-8794

65. Kapeta S, Chondrogianni N, Gonos ES (2010) Nuclear erythroid factor 2-mediated proteasome activation delays senescence in human fibroblasts. J Biol Chem 285(11):8171-8184

66. Papaevgeniou N, Sakellari M, Jha S, Tavernarakis N, Holmberg Cl, Gonos ES et al (2016) 18a-Glycyrrhetinic acid proteasome activator decelerates aging and alzheimer's disease progression in caenorhabditis elegans and neuronal cultures. Antioxid Redox Signal 25(16):855-869

67. Su BN, Jung Park E, Vigo JS, Graham JG, Cabieses F, Fong HH et al (2003) Activity-guided isolation of the chemical constituents of Muntingia calabura using a quinone reductase induction assay. Phytochemistry 63(3):335-341

68. Uda Y, Price KR, Williamson G, Rhodes MJC (1997) Induction of the anticarcinogenic marker enzyme, quinone reductase, in murine hepatoma cells in vitro by flavonoids. Cancer Lett 120(2):213-216

69. Carmona-Gutierrez D, Zimmermann A, Kainz K, Pietrocola F, Chen G, Maglioni $S$ et al (2019) The flavonoid 4,4'-dimethoxychalcone promotes autophagy-dependent longevity across species. Nat Commun 10(1):65

70. Li C, Xu X, Wang XJ, Pan Y (2014) Imine resveratrol analogues: molecular design, Nrf2 activation and SAR analysis. PLoS ONE 9(7):e101455

71. Dinkova-Kostova AT, Massiah MA, Bozak RE, Hicks RJ, Talalay P (2001) Potency of Michael reaction acceptors as inducers of enzymes that protect against carcinogenesis depends on their reactivity with sulfhydryl groups. Proc Natl Acad Sci 98(6):3404

72. Jackson PA, Widen JC, Harki DA, Brummond KM (2017) Covalent modifiers: a chemical perspective on the reactivity of $a, \beta$-unsaturated carbonyls with thiols via Hetero-Michael addition reactions. J Med Chem 60(3):839-885

73. Dinkova-Kostova AT, Talalay P (2010) NAD(P)H:quinone acceptor oxidoreductase 1 (NQO1), a multifunctional antioxidant enzyme 
and exceptionally versatile cytoprotector. Arch Biochem Biophys 501(1):116-123

74. Tsuji PA, Stephenson KK, Wade KL, Liu H, Fahey JW (2013) Structure-activity analysis of flavonoids: direct and indirect antioxidant, and antiinflammatory potencies and toxicities. Nutr Cancer 65(7):1014-1025

75. Chiruta C, Schubert D, Dargusch R, Maher P (2012) Chemical modification of the multitarget neuroprotective compound fisetin. J Med Chem 55(1):378-389

76. Goldman SDB, Funk RS, Rajewski RA, Krise JP (2009) Mechanisms of amine accumulation in, and egress from, lysosomes. Bioanalysis. 1(8):1445-1459

77. Eggler AS, Sergey SN (2013) Chemical and biological mechanisms of phytochemical activation of nrf2 and importance in disease prevention. Recent Adv Phytochem 43:121-155

78. Catherine R-E (2001) Flavonoid antioxidants. Curr Med Chem 8(7):797-807

79. Shanmugam T, Selvaraj M, Poomalai S (2016) Epigallocatechin gallate potentially abrogates fluoride induced lung oxidative stress, inflammation via Nrf2/Keap1 signaling pathway in rats: an in vivo and in silico study. Int Immunopharmacol 39:128-139

80. Ghosh AK, Samanta I, Mondal A, Liu WR (2019) Covalent Inhibition in Drug Discovery. ChemMedChem 14(9):889-906

81. Bandyopadhyay A, Gao J (2016) Targeting biomolecules with reversible covalent chemistry. Curr Opin Chem Biol 34:110-116

82. Vasudevan A, Argiriadi MA, Baranczak A, Friedman MM, Gavrilyuk J, Hobson $A D$ et al (2019) Chapter one-covalent binders in drug discovery. In: Witty DR, Cox B (eds) Progress in medicinal chemistry, vol 58. Elsevier, New York, pp 1-62

83. Hu L, Magesh S, Chen L, Wang L, Lewis TA, Chen Y et al (2013) Discovery of a small-molecule inhibitor and cellular probe of Keap1-Nrf2 proteinprotein interaction. Bioorg Med Chem Lett 23(10):3039-3043
84. Tran KT, Pallesen JS, Solbak SM Ø, Narayanan D, Baig A, Zang J et al (2019) A comparative assessment study of known small-molecule Keap-Nrf2 Protein-protein interaction inhibitors: chemical synthesis, binding properties, and cellular activity. J Med Chem 62(17):8028-8052

85. Pallesen JS, Tran KT, Bach A (2018) Non-covalent small-molecule Kelchlike ECH-associated protein 1-nuclear factor erythroid 2-related factor 2 (Keap1-Nrf2) inhibitors and their potential for targeting central nervous system diseases. J Med Chem 61 (18):8088-8103

86. Zhang P, Zhai Y, Cregg J, Ang KK-H, Arkin M, Kenyon C (2020) Stress resistance screen in a human primary cell line identifies small molecules that affect aging pathways and extend Caenorhabditis elegans'lifespan. Genes Genomes Genetics 10(2):849

87. Kumar V, Kumar S, Hassan M, Wu H, Thimmulappa RK, Kumar A et al (2011) Novel chalcone derivatives as Potent Nrf2 activators in mice and human lung epithelial cells. J Med Chem 54(12):4147-4159

88. Peng S, Zhang B, Meng X, Yao J, Fang J (2015) Synthesis of piperlongumine analogues and discovery of nuclear factor erythroid 2-related Factor 2 (Nrf2) activators as potential neuroprotective agents. J Med Chem 58(13):5242-5255

89. Estrela JM, Mena S, Obrador E, Benlloch M, Castellano G, Salvador R et al (2017) Polyphenolic phytochemicals in cancer prevention and therapy: bioavailability versus bioefficacy. J Med Chem 60(23):9413-9436

\section{Publisher's Note}

Springer Nature remains neutral with regard to jurisdictional claims in published maps and institutional affiliations.
Ready to submit your research? Choose BMC and benefit from:

- fast, convenient online submission

- thorough peer review by experienced researchers in your field

- rapid publication on acceptance

- support for research data, including large and complex data types

- gold Open Access which fosters wider collaboration and increased citations

- maximum visibility for your research: over $100 \mathrm{M}$ website views per year

At $\mathrm{BMC}$, research is always in progress.

Learn more biomedcentral.com/submissions 